\title{
Acesso à saúde sexual e reprodutiva de homens trans em Alagoas
}

\author{
Access to trans men sexual and reproductive health in Alagoas \\ Acceso a la salud sexual y reproductiva hombres trans en Alagoas
}

Recebido: 30/08/2021 | Revisado: 10/09/2021 | Aceito: 14/10/2021 | Publicado: 17/10/2021

Evylee Hadassa Barbosa Silva

ORCID: https://orcid.org/0000-0001-6374-8815 Centro Universitário Tiradentes, Brasil E-mail: evylee.hadassa@ souunit.com.br Joyce Nayara Duarte da Silva

ORCID: https://orcid.org/0000-0002-8728-5648 Centro Universitário Tiradentes, Brasil E-mail:joyce.duarte@souunit.com.br

Alba Maria Bomfim de França

ORCID: https://orcid.org/0000-0001-9474-7137 Centro Universitário Tiradentes, Brasil E-mail: albambf@hotmail.com

\begin{abstract}
Resumo
Verificar como os homens trans em Alagoas acessam os serviços de saúde sexual e reprodutiva em Alagoas. Estudo exploratório, de corte transversal e abordagem quantitativa, sendo utilizada como fonte primária as informações de homens trans relacionadas ao acesso de saúde aos serviços sexuais e reprodutivos de homens trans em Alagoas. Os resultados mostram que, 66,6\% declaram idade entre $18-24$ anos, e 33,3\% entre $25-35$ anos. $40 \%$ possuem ensino superior completo, $40 \%$ ensino médio completo e $20 \%$ ensino superior incompleto. $60 \%$ possuem de $1-3$ salários mínimos, $20 \%$ dispõem de 04 ou mais salário mínimo e os outros $20 \%$ até 01 salário mínimo. $60 \%$ acessam aos serviços de saúde através de plano de saúde particular, e $40 \%$ através do SUS. $60 \%$ não realizaram nenhuma consulta anual nos serviços de saúde sexual e reprodutiva, $20 \%$ realizaram ao menos uma consulta, outros $20 \%$ realizaram 2-4 consultas anualmente. Nenhum dos participantes alegou já ter realizado a cirurgia de redesignação sexual, mas $60 \%$ deles afirmaram que não sentem desejo de fazê-la, outros $40 \%$ sentem o desejo de realizá-la. O acesso à citologia e colposcopia foi relatado por 33,3\%, outros $33,3 \%$ continuam tendo acesso, mas não realizam e, 33,3\% não tem acesso aos serviços de citologia e colposcopia. $\mathrm{O}$ uso de hormonioterapia foi relatado por $80 \%$ dos participantes, já $20 \%$ afirmaram não fazer uso de terapia hormonal. A partir dos resultados obtidos, evidencia-se que o acesso aos serviços de saúde pelos homens trans se dá por meio da rede suplementar de saúde.
\end{abstract}

Palavras-chave: Saúde sexual e reprodutiva; Acesso aos serviços de saúde; Pessoas transgênero.

\section{Abstract}

Check how trans men in Alagoas access sexual and reproductive health services in Alagoas. Exploratory, cross-sectional study with a quantitative approach, using as a primary source information from trans men related to health access to sexual and reproductive services for trans men in Alagoas. The results show that 66.6\% declare age between 18-24 years, and $33.3 \%$ between $25-35$ years. $40 \%$ have completed higher education, $40 \%$ complete high school and $20 \%$ incomplete higher education. $60 \%$ have $1-3$ minimum wages, $20 \%$ have 04 or more minimum wage and the other $20 \%$ up to 01 minimum wage. $60 \%$ access health services through a private health plan, and $40 \%$ through SUS. $60 \%$ had no annual consultations in sexual and reproductive health services, $20 \%$ had at least one consultation, another $20 \%$ had 2-4 consultations annually. None of the participants claimed to have undergone the sex reassignment surgery, but $60 \%$ of them said they do not feel like doing it, another $40 \%$ feel the desire to do it. Access to cytology and colposcopy was reported by $33.3 \%$, another $33.3 \%$ still have access, but do not perform it, and 33.3\% do not have access to cytology and colposcopy services. The use of hormone therapy was reported by $80 \%$ of the participants, while $20 \%$ said they did not use hormone therapy. From the results obtained, it is evident that access to health services by trans men takes place through the supplementary health network.

Keywords: Sexual and reproductive health; Health services accessibility; Transgender persons.

\section{Resumen}

Compruebe cómo los hombres trans en Alagoas acceden a los servicios de salud sexual y reproductiva en Alagoas. Estudio exploratorio, transversal con enfoque cuantitativo, utilizando como fuente primaria información de hombres trans relacionada con el acceso a la salud a servicios sexuales y reproductivos para hombres trans en Alagoas. Los resultados muestran que el 66,6\% declara la edad entre 18-24 años y el 33,3\% entre 25-35 años. El 40\% ha completado la educación superior, el $40 \%$ la secundaria completa y el 20\% la educación superior incompleta. El 60\% tiene 1-3 salarios mínimos, el $20 \%$ tiene 04 o más salario mínimo y el otro $20 \%$ hasta 01 salario mínimo. El 60\% accede a los servicios de salud a través 
de un plan de salud privado y el $40 \%$ a través del SUS. El $60 \%$ no tuvo consultas anuales en los servicios de salud sexual y reproductiva, el $20 \%$ tuvo al menos una consulta, otro $20 \%$ tuvo 2-4 consultas anuales. Ninguno de los participantes afirmó haberse sometido a la cirugía de reasignación de sexo, pero el $60 \%$ de ellos dijo que no tiene ganas de hacerlo, otro $40 \%$ siente el deseo de hacerlo. El 33,3\% reportó acceso a citología y colposcopia, otro 33,3\% aún tiene acceso, pero no lo realiza y el 33,3\% no tiene acceso a servicios de citología y colposcopia. El uso de terapia hormonal fue informado por el $80 \%$ de los participantes, mientras que el $20 \%$ dijo que no usaba terapia hormonal. De los resultados obtenidos se evidencia que el acceso a los servicios de salud por parte de los hombres trans es a través de la red complementaria de salud.

Palabras clave: Salud sexual y reproductiva; Accesibilidad a los servicios de salud; Personas transgénero.

\section{Introdução}

Durante o período pós-II Guerra Mundial, nas décadas de 1950 e 1960, houve um grande aumento populacional, fazendo com que houvesse uma grande preocupação pelo aumento populacional, onde começou-se a haver uma discussão demográficas acerca dos métodos contraceptivos e planejamento reprodutivo, pensando assim, em questões de saúde e direitos reprodutivos (Corrêa, Alves, \& Jannuzzi, 2006).

Recentemente foram estabelecidos os conceitos acerca dos direitos sexuais e reprodutivos, sendo considerado uma conquista histórica, esses direitos foram conquistados através de lutas pela cidadania e pelos direitos humanos. As ações em saúde sexual e reprodutiva tem como marco legal a Conferência Internacional sobre População e Desenvolvimento (CIPD), realizada na cidade do Cairo em 1994, foi determinado que os países realizam esforços para que até o ano de 2015, a saúde reprodutiva estivesse acessível para todos, através do sistema de atenção à saúde (Corrêa, Alves, \& Jannuzzi, 2006).

Apesar disto, a construção dos direitos sexuais e reprodutivos como direitos humanos, ainda esbarram em dificuldades relacionadas às normas morais e aos binarismos homem/mulher, masculino/feminino e heterossexual/homossexual, impedem o acesso de forma integral e a equidade desses direitos em serviços de saúde (Angonese \& Lago, 2017).

No Brasil, a saúde reprodutiva da população trans ainda é bastante negligenciada, um dos motivos para tal é a dificuldade no acesso aos serviços de saúde e as especificidades desta população, onde o atendimento a esses pacientes são marcados pelo preconceito, constrangimento e discriminação, caracterizando assim a presença da transfobia nesses serviços (Angonese \& Lago, 2017).

A não identificação de gênero com o sexo biológico ou a identidade sexual não heteronormativa, traz a população trans a violação de seus direitos humanos básicos, fazendo com que haja uma maior vulnerabilidade. O Ministério da Saúde afirma que a identidade sexual e a identidade de gênero constituem um processo de discriminação e de exclusão, colaborando com os fatores de vulnerabilidade, tais como a violação do direito à saúde (Cardoso \& Ferro, 2012).

A Constituição Federal de 1988 garante a proteção dos direitos humanos no Brasil, apesar das ditas minorias sexuais não serem citadas e da ausência de referências à orientação sexual ou a identidade de gênero, há diversas garantias de direitos a esta população através da Constituição (Brasil, 1988., Angonese \& Lago, 2017).

Diante disto, foi criada a Política Nacional de Saúde Integral de Lésbicas, Gays, Bissexuais, Travestis e Transexuais (LGBT), instituída pelo Ministério da Saúde, por meio da Portaria $\mathrm{n}^{\circ}$ 2.836, de $1^{\circ}$ de dezembro de 2011, garantindo às mulheres transexuais, as travestis e aos homens trans o direito à saúde integral, humanizado e de qualidade no Sistema Único de Saúde (SUS), na rede de atenção básica e em serviços especializados (Ministério da saúde, 2013b).

Esta mesma política define como homens trans aqueles que:

não se identificam com seus genitais biológicos femininos, nem com suas atribuições socioculturais e, em alguns casos podem, através de modificações corporais (hormonioterapia e/ou cirurgia de redesignação sexual), exercer sua identidade de gênero masculina de acordo com seu bem estar biopsicossocial. Identificam-se como homens (identidade de gênero) e podem ser 
heterossexuais, homossexuais e bissexuais (orientação sexual) (Ministério da saúde, 2013b).

Dados obtidos e divulgados pelo Ministério da Saúde, revelam que dois em cada três entrevistados (67\%) já sofreram algum tipo de discriminação ocasionada pela identidade sexual ou pelo gênero, quando analisados os dados de travestis e transexuais esse número alcança cerca de $85 \%$. Já 14,5\% relataram ter sofrido algum tipo de preconceito nos serviços da rede de saúde (Brasil, 2008).

A partir da segunda metade do século XX houve aumento das tecnologias em saúde, principalmente aquelas com finalidades de produzir modificações corporais, essas tecnologias possibilitam que homens trans obtivessem estratégias para modificações corporais, já que essas tecnologias têm potencial de criar o corpo e funcionamentos de gênero desejados (Sousa \& Iriart, 2018).

A saúde da população trans vem se tornando cada vez mais uma pauta relevante e de extrema importância, sendo necessário um maior compromisso do Estado para garantir e responder às demandas desta população que se encontram em grande vulnerabilidade, mas também se caracterizam por serem uma população heterogênea e diversificada (Cardoso \& Ferro, 2012).

Estudo realizado por Sousa e Iriart (2018), identificaram que existem diversas especialidades em que o homem trans procura os serviços de saúde, entre elas estão ginecologistas, endocrinologista, psicóloga/o, clínica/o geral, nutricionista, médica/o cirurgiã/o e outras.

Diante disso, se faz necessário pesquisas acerca desta temática, entendendo aspectos epidemiológicos, sociais, culturais para que seja necessário traçar estratégias para ampliar o acesso desta população aos serviços de saúde em Alagoas. Portanto, a pesquisa tem como objetivo verificar como os homens trans em Alagoas acessam os serviços de saúde sexual e reprodutiva em Alagoas.

\section{Metodologia}

Estudo exploratório, de corte transversal e abordagem quantitativa, sendo utilizada como fonte primária as informações de homens trans relacionadas ao acesso de saúde aos serviços sexuais e reprodutivos de homens trans em Alagoas.

As variáveis em estudo foram divididas em variáveis de caracterização da população (independentes), como idade, procedência, estado civil, filhos, escolaridade, renda média, meio pelo qual acessa serviços de saúde (público ou suplementar); bem como, as variáveis relacionadas ao acesso aos serviços de saúde sexual e reprodutiva: consultas periódicas, acesso a terapias hormonais, acesso a serviços de suporte terapêutico, acesso a cirurgia redesignação sexual, acolhimento por parte da equipe de saúde.

Para fins de coleta de dados, devido a pandemia do novo coronavírus tornou-se inviável a coleta de dados a partir da aplicação do formulário presencial, portanto, optou-se pela aplicação de um formulário online, a partir do Google forms, elaborado para fins deste estudo, tendo como propósito obter informações em relação ao acesso a saúde sexual e reprodutiva de homens trans em Alagoas.

A amostra foi aleatória, tendo em vista que não existe um censo populacional oficial com as informações sobre a população LGBTQIA+, e em especial aos homens trans, em Alagoas. No que se refere aos critérios de inclusão, foram convidados a participar da pesquisa homens transexuais ligados ao conselho LGBT e a Organizações Não-governamentais (ONG) com ações focadas ao referido público, residentes em Alagoas e com idade maior de 18 anos.

Os sujeitos da pesquisa foram convidados a participar do estudo através das redes sociais dos órgãos em questão. Adotou-se como critérios de exclusão homens transexuais portadores de deficiência visual ou outro problema físico ou cognitivo que não permita a leitura do formulário de coleta de dados, em virtude da limitação dos pesquisadores para apresentarem outra forma de 
coleta de dados. Os dados foram analisados a partir de estatística descritiva básica, das frequências absolutas e relativas das respostas sobre o acesso aos serviços de saúde sexual e reprodutiva.

O estudo foi encaminhado ao Comitê de Ética e Pesquisa (CEP), através da Plataforma Brasil, para apreciação, sendo aprovado em 03 de dezembro de 2020 com o número de parecer: 4.436.477 e CAAE: 40200820.5.0000.5641. Os princípios éticos observados por este estudo são, dentre outros, a autonomia, a não maleficência, a beneficência, a justiça e a equidade no intuito de assegurar o anonimato dos participantes do estudo. Foi enviado para os participantes um link contendo o Termo de Consentimento Livre e Esclarecido (TCLE), juntamente com o formulário, ao qual só fora permitido preencher, após a concordância em participar do estudo e o aceite do TCLE, clicando na opção “ACEITO”, destacada no formulário, logo ao final do TCLE.

Os homens trans que se dispuseram a participar do estudo estavam suscetíveis a riscos mínimos como, possível constrangimento ao responder o questionário e quebra do anonimato. Tendo em vista a possibilidade de danos, as pesquisadoras adotaram algumas medidas, para assim, minimizá-los. Aos participantes do estudo, foi assegurado a confidencialidade e a privacidade, a proteção dos dados e as informações não foram utilizadas para prejuízo dos mesmos. Para minimização dos possíveis desconfortos, lhes foi garantida a liberdade de recusa, caso não queiram responder questões que possam lhe causar constrangimento.

No que se refere aos benefícios, mesmo que indiretos, dizem respeito a possibilidade de verificar como os homens trans acessam e são acessados por serviços de saúde sexual e reprodutiva servindo como base para traçar estratégias mais eficazes de acolhida e seguimento das ações de saúde, dessa forma, contribuindo para melhor assistência à saúde. As pesquisadoras comprometeram-se ainda em respeitar os valores culturais, sociais, morais, religiosos e éticos dos participantes do estudo, como também, assumiram a incumbência de divulgar cientificamente os resultados do estudo, sendo possível contribuir para a melhoria das condições de saúde não somente de homens transexuais, mas de toda a comunidade LGBTQIA+.

\section{Resultados e Discussão}

De acordo com os dados obtidos através da pesquisa, ao que se refere o perfil sociodemográfico dos homens trans estudados, como explicitado na Tabela 1, 66,6\% (2) declaram idade entre 18 (dezoito) e 24 (vinte e quatro) anos, 33,3\% (1) declararam ter entre 25 (vinte e cinco) e 35 (trinta e cinco) anos, vale ressaltar que na variável idade apenas 03 (três) homens trans responderam.

Em relação ao estado civil dos participantes do estudo, 20\% (1) afirmam serem solteiros, $40 \%$ (2) casados, 20\% (1) união estável registrada, e 20\% (1) afirmam outro estado civil não abordado nas alternativas do formulário. Todos os participantes alegaram não possuírem filhos. Todos residem na cidade de Maceió-AL.

No quesito da escolaridade dos mesmos, $40 \%$ (2) afirmam que possuem ensino superior completo, $40 \%$ (2) ensino médio completo e $20 \%$ (1) ensino superior incompleto. No tocante à renda dos entrevistados, cerca de 60\% (3) dos participantes da pesquisa dizem possuir de 1 (um) a 3 (três) salários mínimos, 20\% (1) dispõem de 4 (quatro) ou mais salário mínimo e os outros $20 \%$ (1) até 1 (um) salário mínimo. 
Tabela 1 - Caracterização do perfil sociodemográfico de homens transexuais, Alagoas, 2021.

\begin{tabular}{|c|c|}
\hline \multicolumn{2}{|c|}{ Idade* } \\
\hline $\begin{array}{c}18-24 \\
25-35 \\
>35\end{array}$ & $\begin{array}{l}66,6 \%(2) \\
33,3 \%(1) \\
-\end{array}$ \\
\hline \multicolumn{2}{|c|}{ Estado Civil } \\
\hline $\begin{array}{c}\text { Solteiro } \\
\text { Casado } \\
\text { União estável registrada } \\
\text { Outro }\end{array}$ & $\begin{array}{l}20 \%(1) \\
40 \%(2) \\
20 \%(1) \\
20 \%(1)\end{array}$ \\
\hline \multicolumn{2}{|c|}{ Escolaridade } \\
\hline $\begin{array}{l}\text { Ensino Fundamental incompleto } \\
\text { Ensino Fundamental completo } \\
\text { Ensino médio incompleto } \\
\text { Ensino médio completo } \\
\text { Ensino superior incompleto } \\
\text { Ensino superior completo }\end{array}$ & $\begin{array}{c}- \\
- \\
- \\
40 \%(2) \\
20 \%(1) \\
40 \%(2)\end{array}$ \\
\hline \multicolumn{2}{|c|}{ Renda } \\
\hline $\begin{array}{c}\text { Até } 1 \text { salário mínimo } \\
\text { 1-3 salários mínimos } \\
4 \text { ou mais salários mínimos }\end{array}$ & $\begin{array}{l}20 \%(1) \\
60 \%(3) \\
20 \%(1)\end{array}$ \\
\hline \multicolumn{2}{|c|}{ Número de filhos } \\
\hline $\begin{array}{c}\text { Não } \\
\text { Sim, } 1 \text { filho } \\
\text { Sim, } 2 \text { filhos } \\
\text { Sim, } 3 \text { ou mais filhos }\end{array}$ & $\begin{array}{c}100 \%(5) \\
- \\
- \\
-\end{array}$ \\
\hline
\end{tabular}

*Com base em 03 respostas. Fonte: Autores.

Ao que se refere ao acesso aos serviços de saúde pela população pesquisada, através da análise da Tabela 2, foi possível observar que o acesso aos serviços de saúde é predominantemente (60\% ou 03) através de plano de saúde particular, seguido pelo acesso através do SUS (40\% ou 2).

A respeito dos serviços de saúde sexual e reprodutiva, 50\% (2) dos alagoanos estudados possuem acesso a estes serviços pelos planos de saúde, e 50\% (2) não possuem acesso a nenhum tipo de serviço de saúde sexual e reprodutiva, vale ressaltar que apenas 04 (quatro) indivíduos responderam a essa pergunta.

Quanto ao acolhimento por parte das equipes de saúde, $20 \%$ (1) alegaram que o seu nome social não foi respeitado, em contrapartida, $80 \%$ (4) afirmaram que foram bem acolhidos e respeitados em todos os momentos da assistência.

No tocante a quantidade de consultas anuais nos serviços de saúde sexual e reprodutiva, 60\% (3) dos homens trans estudados não realizaram nenhuma consulta, $20 \%$ (1) realizou ao menos uma consulta, outros $20 \%$ (1) realizou de duas a quatro consultas anualmente.

Sobre o acesso à serviço de suporte terapêutico, $50 \%$ (2) dos homens estudados afirmaram ter acesso a um serviço de suporte terapêutico por meio de um psicólogo, 25\% (1) afirmaram ter acesso a esse serviço por meio de outras terapias, e outros $25 \%$ (1) informaram não ter acesso a nenhum suporte terapêutico, vale lembrar que essa variável foi respondida por apenas 04 indivíduos. 
Tabela 2 - Acesso ao serviço de saúde por homens trans, Alagoas, 2021.

\begin{tabular}{|c|c|}
\hline \multicolumn{2}{|c|}{ Acesso os serviços de saúde em geral } \\
\hline $\begin{array}{l}\text { SUS } \\
\text { Plano de Saúde } \\
\text { Consultas pagas } \\
\text { Não acessa }\end{array}$ & $\begin{array}{l}40 \%(2) \\
60 \%(3) \\
- \\
-\end{array}$ \\
\hline \multicolumn{2}{|c|}{ Acesso aos serviços de saúde sexual e reprodutiva ${ }^{* *}$} \\
\hline $\begin{array}{l}\text { Pelo SUS } \\
\text { Pelo plano de saúde } \\
\text { Consultas pagas } \\
\text { Não acesso }\end{array}$ & $\begin{array}{c}- \\
50 \%(2) \\
- \\
50 \%(2)\end{array}$ \\
\hline \multicolumn{2}{|c|}{ Acesso à serviço de suporte terapêutico** } \\
\hline $\begin{array}{l}\text { Sim. Psicólogo } \\
\text { Sim. Grupos de apoio } \\
\text { Sim. outras terapias } \\
\text { Não }\end{array}$ & $\begin{array}{l}50 \%(2) \\
- \\
25 \%(1) \\
25 \%(1)\end{array}$ \\
\hline \multicolumn{2}{|c|}{ Acolhimento por parte das equipes de saúde } \\
\hline $\begin{array}{l}\text { Meu nome social não foi respeitado } \\
\text { Fui vítima do preconceito } \\
\text { Fui bem acolhido e respeitado em todos os momentos } \\
\text { da assistência }\end{array}$ & $\begin{array}{c}20 \%(1) \\
- \\
80 \%(4)\end{array}$ \\
\hline \multicolumn{2}{|c|}{ Número de consultas anuais nos serviços de saúde sexual e reprodutiva } \\
\hline $\begin{array}{c}\text { Nenhuma } \\
1 \text { consulta } \\
2-4 \text { consultas } \\
5 \text { ou mais consultas }\end{array}$ & $\begin{array}{l}60 \%(3) \\
20 \%(1) \\
20 \%(1) \\
\quad-\end{array}$ \\
\hline
\end{tabular}

*Com base em 4 respostas. Fonte: Autores.

Em relação à possibilidade da realização da cirurgia de redesignação sexual, ao serem questionados sobre a realização do procedimento, os participantes que responderam que não o realizaram, foram direcionados a responder mais duas perguntas, $o$ conjunto de respostas, está apresentado na Tabela 3. A qual, exibe que nenhum dos participantes alegou já ter realizado a cirurgia, mas $60 \%$ (3) deles afirmaram que não sentem desejo de fazê-la, outros $40 \%$ (2) sentem o desejo de realizá-la.

Tendo em vista que, as genitálias femininas e estruturas reprodutivas permanecem em homens trans caso não haja a realização da cirurgia de redesignação sexual, foi sondado através do formulário se ainda havia o acesso à citologia e colposcopia, e, 33,3\% (1) deles afirmaram continuar tendo acesso a citologia e colposcopia e realizar no prazo devidamente, 33,3\% (1) alegaram continuar tendo acesso a citologia e colposcopia, mas não realizam, 33,3\% (1) afirmaram não ter acesso aos serviços de citologia e colposcopia, vale acentuar que apenas 03 (três) indivíduos responderam a essa pergunta.

Com respeito ao uso terapia hormonal, $80 \%$ (4) afirmaram o uso de alguma terapia hormonal prescrita por profissionais de saúde, já 20\% (1) afirmaram não fazer uso de nenhuma terapia com hormônios. 
Tabela 3 - Acesso a serviços/demandas de saúde específicas à saúde sexual e reprodutiva por homens trans, Alagoas, 2021.

\section{Cirurgia de redesignação sexual}

\begin{tabular}{|c|c|}
\hline $\begin{array}{l}\text { Não realizei, mas desejo } \\
\text { Já realizei } \\
\text { Não realizei, e não desejo }\end{array}$ & $\begin{array}{c}40 \%(2) \\
- \\
60 \%(3)\end{array}$ \\
\hline \multicolumn{2}{|c|}{ Acesso/realização de consultas/procedimentos/exames ginecológicos* } \\
\hline $\begin{array}{l}\text { Continuo tendo acesso a citologia e colposcopia e } \\
\text { realizo no prazo devidamente }\end{array}$ & $33,3 \%(1)$ \\
\hline $\begin{array}{l}\text { Continuo tendo acesso a citologia e colposcopia, mas } \\
\text { não realizo }\end{array}$ & $33,3 \%(1)$ \\
\hline $\begin{array}{l}\text { Não tenho acesso aos serviços de citologia e } \\
\text { colposcopia }\end{array}$ & $33,3 \%(1)$ \\
\hline \multicolumn{2}{|c|}{ Uso de terapia hormonal } \\
\hline Sim. Prescrito por profissionais de saúde & $80 \%(4)$ \\
\hline Sim. Por conta própria/indicação de amigos/internet & - \\
\hline Não & $20 \%(1)$ \\
\hline
\end{tabular}

*Com base em 3 respostas. Fonte: Autores.

Em relação aos dados sociodemográficos apresentados de homens trans estudados em Alagoas, verificou-se que o mesmo é predominantemente de alta escolaridade e que recebem até 3 salários mínimos, a faixa etária é de 18 a 25 anos, casados, e não possuem filhos. A pesquisa realizada por Solka e Antoni (2020), demonstrou o mesmo grau de escolaridade entre os homens trans, onde a maioria possuíam ensino médio e ensino superior completo/incompleto, corroborando com os dados aqui encontrados. A faixa etária foi semelhante a um estudo que teve como objetivo analisar a diversidade e o perfil sociodemográfico da população trans / travesti (Carrara et al., 2019). Ainda, no presente estudo, a maioria dos participantes afirmaram ter parceiro(a) fixo(a), sendo $40 \%$ casados e $20 \%$ vivem em união estável, ao contrário do que demonstrou o estudo de Tornello \& Bos (2017), onde 45,8\% do total de homens trans declarou estado civil como solteiro.

Apesar de todos os indivíduos participantes desta pesquisa afirmarem não possuir filhos, engravidar e ter filhos tem se tornado uma realidade cada vez mais comum para homens trans (Pinho, Rodrigues, \& Nogueira, 2020). Com as diversas mudanças sociais e legais em relação aos indivíduos transgênero, se tem percebido uma ampliação na discussão sobre a paternidade dessa população (Tornello \& Bos, 2017). Como apontou um estudo de revisão da literatura, que analisou pesquisas existentes sobre a prevalência e as características de pessoas trans que são pais, 25\% - 50\% dos transexuais atualmente possuíam filhos (Stotzer, Herman, \& Hasenbush, 2014).

Os direitos sexuais e reprodutivos são considerados uma ampliação dos direitos humanos, onde é possível garantir de forma integral a liberdade e a autonomia em relação ao corpo. Partindo desta definição, o reconhecimento desses direitos é de extrema importância para o desenvolvimento e ampliação de políticas públicas de saúde que abranjam as especificidades e individualidade de cada população, incluindo a população transexual (Angonese \& Lago, 2017).

Segundo estudo realizado nos Estados Unidos, que teve como objetivo investigar o impacto da exclusão e estigma na utilização de serviços de saúde, que apontou para altos índices nos escores nas escalas de estigma tiveram relação direta com menor acesso e uso dos serviços de saúde para a população transgênero. Sendo obtidos através da amostra total que, 37,0\% não possuíam acesso aos serviços de saúde, em relação aos que frequentavam, 43,0\% relataram que sua orientação sexual ou identidade de gênero não afetava sua saúde e 27,0\% alegaram que esta informação não era levada em consideração pelos profissionais de saúde, gerando 
assim, uma assistência fragmentada (Whitehead, Shaver, \& Stephenson, 2016).

De acordo com o Caderno de Atenção básica n 26, que diz respeito à saúde sexual e reprodutiva, as intervenções direcionadas à saúde sexual e reprodutiva são, majoritariamente, concentradas na reprodução, tendo como foco a mulher em sua idade fértil/adulta, assim, postergando o empenho na promoção de ações de saúde voltadas para os homens de uma forma geral. Tal cenário na realidade brasileira, é refletido nos resultados encontrados no presente estudo, tanto no que toca a quantidade de consultas anuais, visto que a maioria (60\%) não teve sequer uma consulta a esses serviços no período de um ano, quanto no acesso a esses serviços, pois 50\% (2) dos indivíduos estudados não têm acesso algum à esses serviços (Ministério da saúde, 2013a).

Outro serviço de importância ímpar no processo da transexualidade é o suporte terapêutico. O estudo realizado por Vieira e Porto (2019), levanta uma reflexão pertinente sobre a alta adesão ao suporte terapêutico ofertado por psicólogos em relação às outras terapias, como foi observado no presente estudo através da Tabela 2. A pesquisa traz à tona o fato de um interesse específico no comparecimento às consultas ao psicólogo e às consultas médicas, por conta da viabilidade das receitas legais para uso de hormônios.

No que tange o respeito ao nome social de homens trans, a literatura relata que a ausência na garantia do direito e respeito do nome social escolhido pela população transexual e travesti, é caracterizado como violência, gerando constrangimento, sofrimento e dificulta o acesso aos serviços de saúde, o que fere os direitos do usuário e os princípios fundamentais do SUS, baseados na universalidade do acesso, equidade, integralidade e humanização da assistência (Silva, Silva, Coelho \& Martiniano, 2017).

Apesar do que demonstra a literatura e pesquisas realizadas, os resultados desta pesquisa demonstraram que a maioria (80\%) dos participantes tiveram o seu nome social respeitado e utilizado durante a assistência de saúde. O que vai de encontro ao estudo realizado por Rocon, Rodrigues, Zamboni, e Pedrini (2016) onde os participantes afirmam que o desrespeito ao nome social e outros acontecimentos nos serviços de saúde é um fator crucial para não efetivação do acesso a serviços essenciais.

O Brasil é marcado por diversas desigualdades sociais, gerando acesso limitado aos direitos básicos da população, recebendo destaque alguns marcadores sociais da diferença, tais como gênero, orientação sexual, classe social e etnia/raça, o que favorece a vulnerabilidade sociais, econômicas, culturais e políticas para determinados grupos. Ao que se refere a população LGBTQIA+, a junção destes marcadores realça a discriminação, corrobora a exclusão e complexifica o acesso aos serviços de saúde, educação e trabalho (Oliveira, Nogueira, Costa, Silva, \& Almeida, 2018).

Nenhum dos participantes desta pesquisa alegaram terem realizado a cirurgia de redesignação sexual, sendo apresentado pela maioria (60\%) o não interesse pela cirurgia, já o interesse pela cirurgia foi apresentado por $40 \%$ dos participantes. No estudo realizado por Kailas, Lu, Rothman, e Safer, (2017), que teve como objetivo avaliar a prevalência de cirurgias de afirmação de gênero entre pacientes transgêneros em 2015, demostrando que cerca 35\% da amostra total dos indivíduos haviam realizado pelo menos uma cirurgia de afirmação de gênero.

A literatura aponta que, as mudanças corporais são processos realizados com o intuito de homens trans encontrarem o corpo idealizado, onde na maioria dos casos, está relacionado a uma referência do corpo cisgênero masculino. Essa estratégia visa a reprodução do gênero com o intuito de viabilizar o reconhecimento e a aceitação em diversos âmbitos (Sousa \& Iriart, 2018). O procedimento cirúrgico transexualizador é tido como um importante serviço para a população trans que almejam intervenções cirúrgicas e hormonais, no entanto, este é um processo em que o sentido não deve ser apenas centrado nas cirurgias de redesignação sexual (Rocon et al., 2020).

No que concerne ao acesso aos serviços de citologia e colposcopia pelos homens trans desta pesquisa, é notório que ainda há uma grande inadequabilidade desse acesso, o que representa 33,3\% (1) dos indivíduos da pesquisa. Um estudo realizado por Harb, Pass, Soriano, Zwick, e Gilbert (2019), indicou que 59,0\% da amostra total da pesquisa haviam tido acesso aos mesmos serviços de saúde, o que demonstra uma diferença significativa com os resultados desta pesquisa. Esta mesma pesquisa ainda 
revelou que os participantes tinham conhecimento sobre o papilomavírus humano (HPV) e os exames de Papanicolaou, mas relataram não estarem bem informados sobre o HPV.

Tendo em vista diversos fatores, grande parte da população de homens trans não realizam ou não possuem o desejo de realizar a redesignação sexual, sendo assim, continuam expostos aos fatores de risco para o desenvolvimento do câncer de colo do útero, se fazendo necessário a adoção de medidas preventivas como exames preventivos e imunizações (Peitzmeier, Reisner, Harigopal, \& Potter, 2014). No Brasil, o rastreamento do câncer do colo do útero é realizado através do exame citopatológico (exame de Papanicolau), este método está disponível para mulheres e qualquer outra pessoa que possua o colo do útero na faixa etária 25 a 64 anos e que tenham começado a atividade sexual (Instituto Nacional de Câncer José Alencar Gomes da Silva, 2016). Incluindo homens trans e pessoas não binárias denominadas mulheres ao nascer (Connolly, Hughes, \& Berner, 2020).

Ao que se refere o uso de hormonioterapia, a maioria dos participantes (80\%) alegaram fazer uso de algum tipo de terapia hormonal prescrita por profissional de saúde. A hormonioterapia é conhecida há cerca de um século, sendo uma das alternativas utilizada por homens trans para tratar a disforia de gênero, onde a literatura científica traz ampla evidência da eficácia desse tipo de intervenção (Gorton \& Erickson-Schroth, 2020). Um estudo de revisão sistemática e meta-análise realizado em 2010, apontou que o uso de tratamento hormonal em $86 \%$ dos homens trans tiveram resultados bastantes positivos em relação à disforia de gênero, e 78\% apresentou melhoria significativa na qualidade de vida (Murad et al, 2010).

O processo transexualizador tem sido cada vez mais discutido na literatura científica, onde os serviços de saúde especializado para tal processo ainda é notório a ausência de regulamentação e cuidado com a prescrição, o uso de hormônios e seu acompanhamento. Essas questões podem ser ainda mais perceptíveis aos homens trans, onde o acesso a testosterona é dificultado pelo fato de ser um hormônio altamente regulamentado (Lima \& Cruz, 2016). Apesar das dificuldades apontadas, foi verificado no presente estudo que $80 \%$ dos homens trans participantes fazem uso de hormonioterapia.

\section{Conclusão}

A partir dos resultados obtidos, evidencia-se que o acesso aos serviços de saúde pelos homens trans se dá por meio da rede suplementar de saúde. Já no que diz respeito à caracterização sociodemográfica, observa-se que se tratam de adultos jovens, com parceiros, grau de escolaridade entre o ensino médio e o superior, renda média entre 1 e 3 salários mínimos vigentes e sem filhos. O acolhimento por parte da equipe de saúde é bastante positivo, onde a maioria dos homens trans alegaram que foram bem acolhidos e respeitados em todos os momentos da assistência.

\section{Referências}

Angonese, M. \& Lago, M. C. S. (2017). Direitos e saúde reprodutiva para a população de travestis e transexuais: abjeção e esterilidade simbólica. Saúde e Sociedade. $26(1), 256-270$.

Carrara, S., Hernandez, J. G., Uziel, A. P., Conceição, G. M. S., Panjo, H., Baldanzi, A. C. O., Queiroz, J. P., D’Angelo, L. B., Balthazar, A. M. S., Junior, A. L. S., \& Giami, A. (2019). Body construction and health itineraries: a survey among travestis and trans people in Rio de Janeiro, Brazil. Caderno de Saúde Pública. 35(4), $1-15$

Cardoso, M. R. \& Ferro, L. F. (2012). Saúde e população LGBT: demandas e especificidades em questão. Psicologia: ciência e profissão. 32 (3), $552-563$.

Constituição (1988). Constituição da República Federativa do Brasil 1988. (1988). Senado Federal: Centro Gráfico, 1988.

Corrêa, S; Alves, J. E. D; Jannuzzi, P. M. (2006). Direitos e saúde sexual e reprodutiva: marco teórico-conceitual e sistema de indicadores. Indicadores municipais de saúde sexual e reprodutiva. (1) 27-62.

Connolly, D., Hughes, X., \& Berner, A. (2020). Barriers and facilitators to cervical cancer screening among transgender men and non-binary people with a cervix: A systematic narrative review. Preventive Medicine. (135), 1-34.

Gorton, R. N. \& Erickson-Schroth, L. (2020). Hormonal and Surgical Treatment Options for Transgender Men (Female-to-Male). Psychiatr Clin North Am. 40 (1), 79-97. 
Harb, C. Y. W., Pass, L. E., Soriano, I. C., Zwick, A., \& Gilbert, P. A., (2019). Motivators and Barriers to Accessing Sexual Health Care Services for Transgender/Genderqueer Individuals Assigned Female Sex at Birth. 4 (1), 58-67.

Instituto Nacional de Câncer José Alencar Gomes da Silva. Coordenação de Prevenção e Vigilância (2016). Divisão de Detecção Precoce e Apoio à Organização de Rede. Diretrizes brasileiras para o rastreamento do câncer do colo do útero. Revista atual. 2. ed.

Kailas, M., Lu, H. M. S., Rothman, E. F., \& Safer, J. D. (2017). Prevalence and types of gender-affirming surgery among a sample of transgender endocrinology patients prior to state expansion of insurance coverage. Endocr Pract. 23 (7), 780-786.

Lima, F. \& Cruz, K. T. (2016). Os processos de hormonização e a produção do cuidado em saúde na transexualidade masculina. Sexualidad, Salud y Sociedad Revista Latino Americana.. (23), 162-186.

Ministério da Saúde. (2013a). Cadernos de Atenção Básica, n. 26. Ministério da Saúde.

Ministério da Saúde. (2013b) Política Nacional de Saúde Integral de Lésbicas, Gays, Bissexuais, Travestis e Transexuais. Ministério da Saúde.

Ministério da Saúde (2008). Saúde da população de gays, lésbicas, bissexuais, travestis e transexuais. Revista Saúde Pública, 42 (3), 570-573. Departamento de Apoio à Gestão Participativa, Secretaria de Gestão Estratégica e Participativa. Brasília, DF: Ministério da Saúde.

Murad, M. H., Elamin, M. B., Garcia, M. Z., Mullan, R. J., Murad, A., Erwin, P. J., \& Montori, V. M. (2010). Hormonal therapy and sex reassignment: a systematic review and meta-analysis of quality of life and psychosocial outcomes. Clinical Endocrinology. 72 (2), 214-231.

Oliveira, G. S., Nogueira, J. A., Costa, G. P., Silva, F. V., \& Almeida, S. A. (2018). Access by lesbians, gays, bisexuals and transvestites/transsexuals to the Basic Family Health Units. Revista Rene. 19, 1-7.

Peitzmeier, S. M., Reisner, S. L., Harigopal, P., \& Potter, J. (2014). Pacientes do sexo feminino para masculino têm alta prevalência de Papanicolaou insatisfatório em comparação com mulheres não transgênero: implicações para o rastreamento do câncer cervical. Journal of general internal medicine. 29 (5), 778-784.

Pinho, A. R., Rodrigues, L., \& Nogueira, C. (2020). (DES)Construção da parentalidade trans*: homens que engravidam. Ex cequo. 41, 195-205.

Rocon, P. C., Rodrigues, A., Zamboni, J., \& Pedrini, M. D. (2016). Dificuldades vividas por pessoas trans no acesso ao Sistema Único de Saúde. Ciência \& Saúde Coletiva. 21 (8), 2517-2526.

Rocon, P. C., Sondré, F., Rodrigues, A., Barros, M. E. B., Pinto, G. S. S., \& Roseiro, M. C. F. B. (2020). Life after sexual reassignment surgery: significance for gender and transsexuality. Ciência \& Saúde Coletiva. 25 (6), 2347-2356

Silva, L. K. M., Silva, A. L. M. A., Coelho, A. A., \& Martiniano, C. S. (2017). Uso do nome social no Sistema Único de Saúde: elementos para o debate sobre a assistência prestada a travestis e transexuais. Physis: Revista de Saúde Coletiva. 27 (3), 835-846.

Sousa, D. \& Iriart, J. (2018). "Viver dignamente": necessidades e demandas de saúde de homens trans em Salvador, Bahia, Brasil. Caderno de Saúde Pública. 34 (10), 1-11.

Solka, A. C. \& Antoni, C. (2020). Homens trans: da invisibilidade à rede de atenção em saúde. Revista Saúde e Desenvolvimento Humano. 8 (1), 7-16.

Stotzer, R. L., Herman, J. L., Hasenbush, A. (2014). Transgender parenting: a review of existing research. The Williams Institute. 1-27.

Tornello, R. L. \& Bos, H. (2017). Parenting Intentions Among Transgender Individuals. LGBT Health. 4 (2), 1-6.

Vieira, C. \& Porto, R. M. (2019). "Fazer emergir o masculino": noções de "terapia" e patologização na hormonização de homens trans. Cadernos Pagu. (55), 1-32.

Whitehead, J., Shaver, J., \& Stephenson, R. (2016). Outness, Stigma, and Primary Health Care Utilization among Rural LGBT Populations. Journal pone. 11 (1.) 